\title{
Visualization Study and Quantitative Velocity Measurements in Turbulent Taylor-Couette Flow by Phantomm Flow Tagging: a Description of the Transition to Turbulence
}

\author{
J. C. C. Campos \\ Polytechnic School of Uberlândia \\ Fernando Vilela, 839 \\ 38400-456 Uberlândia, .MG. Brazil \\ jcampos@uber.com.br
}

A visualization study and Quantitative velocity measurements have been performed in Taylor-Couette flow with a medium-gap $(c=0.356)$, over a large range of Taylor numbers $\left(2.1 \times 10^{4}<T a<1.1 \times 10^{11}\right)$, with the outer cylinder fixed and the inner cylinder rotating about its axis. Quantitative velocity measurements were carried out using the PHANTOMM flow tagging technique. Two techniques were used for visualization study: the PHANTOMM technique that allowed flow structure visualization from small to moderate Taylor numbers, and the Particle Streak Imaging, PSI (the flow was seeded with neutrally buoyant polystyrene micro spheres) that permitted the flow structure visualization from moderate to high Taylor numbers. The results illustrate the expected three-dimensional features of flow and presence of Taylor cells at low Taylor numbers. Our study examined the interplay between small and large scales present in the flow as well as showed the gradual transition to turbulence with increasing Taylor numbers. Taylor cells were found for Taylor numbers less than $1.13 \times 10^{10}$. At low Taylor numbers, the flow in the cells appeared to be a rotational laminar flow with a high degree of coherence. At higher Taylor numbers, the cells aspect became more irregular, and the flow inside them became turbulent. The Görtler instability developed inside Taylor cells and close to the inner cylinder wall. At the highest Taylor numbers, turbulence increased up to the point where no Taylor cells could be detected. For the flow in our study, at Ta $=1.13 \times 10^{10}$, the homogenization by turbulence spread across the gap, and the flow structure sharply changed its pattern as a toroidal vortex in helical motion developed in a thin layer on the inner cylinder wall. Instantaneous velocities, average velocities, angularmomentum ratio and spectral density function were computed for all ranges of Taylor numbers in the range studied. These quantitative results show the same conclusions as the ones presented by the visualization study.

Keywords: Visualization study, turbulences, Taylor-Couette flow, phantom, flow tagging

\section{Introduction}

\section{The Taylor-Couete Flow and Objectives}

A particularly interesting aspect of the Taylor-Couette flow study is the transition from laminar to turbulent flow. Studies of instabilities at Taylor numbers that manifest themselves slightly higher than the critical value for the onset of Taylor vortices were performed by Ioos (1986); and Benjamin \& Mullin (1982), among many others, but the complete transition to the turbulence takes place at Taylor numbers higher than those studied in previous papers. In fact, most of the earlier studies were carried out at Taylor numbers lower than the ones corresponding to that expected for a complete transition. A possible exception is the paper of Smith \& Townsend (1982) who studied Taylor-Couette flow for Taylor numbers larger than the other authors, but they did not visualize the flow structure.

Some of the studies in Taylor-Couette flow have suggested that the flow structure is composed of large Taylor cells throughout the gap, typically due to the Taylor instability (also called the instability of the first kind) and an instability characteristic of the Görtler instability (also called either a secondary instability or an instability of the second kind) in the thin boundary layer on the inner cylinder wall. The disturbances in the boundary layer are of the counter-

Paper accepted October, 2003. Technical Editor: Aristeu da Silveira Neto. rotating vortex pairs form, with axes in the circumferential direction, with a motion similar of that Taylor cell (Smith \& Townsend 1982; Barcilon \& Brindley 1984; Wei et al. 1992). It should be noted that the Görtler instability is not limited to concave wall geometry. For example, this instability also occurs in a wall jet over a convex surface, and Görtler vortex structures exist in turbulent boundary layers over concave surfaces such as the pressure-side of turbinecompressor blades (Floryan 1991).

The Görtler instability may also be an important mechanism for the transition control in a boundary layer along curved surfaces, for both compressible and incompressible turbulent flow. One of the first investigations concerned with the Görtler instability in compressible flow was due to Ginoux (1971) who observed the presence of Görtler vortices in the re-attaching flow downstream of a backward-facings step at a Mach number of 5.5. The experimental evidence shows

that the boundary layer becomes unstable, leading to Görtler vortices. These vortices were also observed in the presence of a shock wave boundary layer interaction (Delery \& Coet 1985). In addition, high noise levels - which are caused by Mach wave radiation from the turbulent boundary layers on the nozzle walls - in conventional supersonic wind tunnels cause premature transition from laminar to turbulent flow in the boundary layers on test models (Chen et al. 1985). An important step in delaying transition is to control the amplification of the Taylor-Görtler vortices. It is known that the transition of a boundary layer from laminar to turbulent flow on the wall of a supersonic nozzle can also be caused by a Görtler instability. 
Turbulence in Taylor-Couette flow may be better understood if one thinks that the transition process in turbulence is guided by two mechanisms. The first one is led by the overall structure, that is, the Taylor cells. These cells present slower changes and contain intermittent turbulent flow at high Taylor number. If Taylor cells are present in highly turbulent flow, they set up particular characteristics to the turbulence linked with the system geometry and provided with a characteristic time scale. The second process is linked with the transition mechanism on a small scale (Görtler instability). This mechanism exists within the Taylor cell that becomes turbulent at high Taylor number.

Some of the previous studies have visualized the flow by the front lighting of a radial plane and by seeding the flow with the reflecting particles (Coles 1965;Fenstermacler et al. 1979). This visualization method has been inadequate to show the small-scale, counter-rotating vortices formation (Görtler instability). It has also been inadequate to show the large-scale structure (Taylor cell) at high Taylor number. Generally, experimental techniques applied to Taylor-Couette flow were designed to provide an evidence that small-scales lead flow either to turbulence (Wei et al. 1992) or to find the point that corresponds to the critical Taylor number in the lower Taylor number flow (Coles 1965). However, the interplay between small and large scales in a gradual transition to turbulence at high Taylor numbers is not well understood.

It is due to such reasons that we have made a new study of Taylor-Couette flow. Our primary objective was to study the Taylor cells structure, and the instabilities in the boundary layer on the inner cylinder, over the Taylor number range where the flow goes from laminar to turbulent. In particular, we aimed:

I. to determine the interplay between small and large scales in a gradual transition to turbulence, and

II. to determine if Taylor cells are present in highly turbulent flow, and, if so, to study their stability: how they influence the turbulence behavior, and how they depend on the system geometry.

In addition, the aspects of the Taylor-Couette flow described in the paragraph above have not been investigated in details by quantitative velocity measurements. The hot wire anemometer, by Smith \& Townsend (1982), is not adequate for this study because it affects adversely the flow structure within the small gaps. Also, it is not possible to get information about the large Taylor cells global features since it is inherently a single-point technique. This aspect may also restrict the LDV (Laser Doppler Anemometry) use. On the other hand, the PHANTOMM technique can extract defined interesting facts of the flow structure because spatial measurements performed with this technique provide information what happens along a line. The statistical treatment of velocities of spatial changes can help to show the main flow aspects.

Thus, we opted for studying the Taylor-Couette flow using the PHANTOMM technique. In this investigation by quantitative velocity measurements, we aim the following objectives:

I. To characterize the performance this technique presented to measure velocities in incompressible turbulent flow in high Reynolds number, and

II. To carry out a measurements treatment with an aim to understand the flow structure as a function of Taylor number, in the range where the flow goes from laminar to turbulent. The measurement procedure used then will be explained in the next section.

\section{The Phantomm Technique}

The ability to obtain accurate, high spatial resolution velocity data over a wide range of Reynolds numbers poses a significant challenge to existing optical diagnostic techniques. Flow tagging is an optical diagnostic in which a laser beam is used to "write" a spatially continuous pattern into a specific flow field region. The temporal evolution of the initial pattern is tracked ("interrogated") through Laser-Induced Fluorescence (LIF) imaging. The displacement within the elapsed time interval constitutes a measurement of velocity, with an absolute accuracy limited only by the ability to determine position and time.

Flow tagging based on caged dye Photo-Activated Fluorphores (PAF) (McCray 1989). PAF tracers has been termed as PhotoActivated Nonintrusive Tracking of Molecular Motion (PHANTOMM). Caged dye PAF's are organic dye molecules in which a chemical caging group has been attached in order to quench the normally bright laser fluorescence. The caging group is photolytically cleaved upon an exposure of the wazzu molecule to ultraviolet (UV) light, typically, but not necessarily, from a laser. Upon photolysis, the original dye is recovered which can be tracked indefinitely using ordinary laser sheet fluorescence imaging approaches (Dahm et al. 1990). As was discussed by Lempert et al. (1995), the uncaged dye exhibits an extremely intense fluorescence, with the result that low concentrations (less than $10^{-6} \mathrm{M}$ ) are required. As in ordinary dye visualization, the fluorescence is Stokes-shifted so that simple long-pass colored glass filters can be used to attenuate elastic scattering from the laser. This produces high contrast images which are conducive to measurements at high Reynolds number. In many respects, the technique is similar to Laser-Induced Photochemical Anemometry (LIPA), in which time lines are written into a flow using photochromic phosphorescent materials (Falco \& Nocera 1993).

The technique capabilities for measuring velocities in highspeed flow are directly linked to PAF tracers optical and chemical properties. In particular, it should be pointed out that, while there is no limit to maximum time between cage-breaking (tag) and interrogation (since the photochemical change is permanent), there is a minimum time dictated by the kinetic rate for the cage-breaking photolysis process. The finite rate results in a time lag between the firing of the tagging laser and the evolution of sufficient dye in its fluorescent form to be interrogated with sufficient signal-to-noise. The magnitude of the signal-to-background ratio is a function of the caged dye purity, as well used as the sheet thickness. This ratio needs to be adjusted by experiment (Lempert et al. 1995).

\section{Concepts and Definitions}

For a cylindrical geometry, $(\mathrm{r}, \theta, \mathrm{z})$ to represent the radial, azimuthal and vertical axial directions, respectively, and the basicstate velocity vector $\vec{V}=\left(v_{r}, v_{\theta}, v_{z}\right)$, is represented by radial, tangential and axial velocity components. Rayleigh (1916) showed that the necessary and sufficient condition for the existence of an inviscid axisymmetric instability is:

$$
\frac{\mathrm{d}\left(\Gamma^{2}\right)}{\mathrm{dr}}<0
$$

anywhere in the flow. $\Gamma$ is the circulation defined as $\Gamma=r V_{\theta}$.

The instability in Taylor-Couette flow is governed by the Taylor Number, $T_{\mathrm{a}}$, defined by

$$
\begin{array}{r}
\mathrm{Ta}=\mathrm{Re}^{2} \delta, \quad \mathrm{Re}=\frac{\mathrm{V}_{\mathrm{i}} \mathrm{d}}{v}, \quad \mathrm{~V}_{\mathrm{i}}=\mathrm{R}_{\mathrm{i}} \Omega_{\mathrm{i}} \\
\mathrm{d}=\mathrm{R}_{\mathrm{o}}-\mathrm{R}_{\mathrm{i}}, \delta=\frac{\mathrm{d}}{\overline{\mathrm{r}}}\left(1-\mu^{2}\right), \overline{\mathrm{r}}=\frac{\mathrm{R}_{\mathrm{i}}+\mathrm{R}_{\mathrm{o}}}{2}
\end{array}
$$

\section{J. of the Braz. Soc. of Mech. Sci. \& Eng. Copyright () 2003 by ABCM October-December 2003, Vol. XXV, No. 4 / 379}




$$
\mu=\frac{\Omega_{\mathrm{o}}}{\Omega_{\mathrm{i}}}
$$

where $v$ is the cinematic viscosity, $R_{i}$ and $R_{o}$ are the radii of the inner and outer cylinders, respectively, $\Omega_{\mathrm{i}}$ and $\Omega_{\mathrm{o}}$ and are the inner and outer cylinders angular velocities.

When the outer cylinder is fixed and the inner cylinder is rotating about its axis $\mu=0$. This case is the one studied in this paper.

The parameter that determines the establishment of the Görtler vortex instability is the Görtler number defined by Eq. (9). In this equation, $V_{\infty}$ is the local free-stream velocity, $\delta$ is the boundarylayer thickness and $\bar{r}$ is the local mean radius of curvature, defined by Eq. (7).

$$
\mathrm{G}=\frac{\mathrm{V}_{\infty} \delta}{v}\left(\frac{\delta}{\overline{\mathrm{r}}}\right)
$$

The Görtler number is proportional to the square root of Taylor number. Eqs. (2) and (9) establish an analogy between TaylorCouette and boundary layer flow on a concave surface. Experiments in Taylor-Couette may have important consequences for the boundary layer control on concave surfaces.

Most of discussions on Taylor-Couette flow have concentrated on cases with small gaps, although important changes occur when the gap size increases (Stuart 1986). The parameter that characterizes the gap length is the radius ratio, $\eta$, by Eq. (10):

$$
\eta=\frac{R_{i}}{R_{o}}
$$

In general, we can classify the gap size as follows:

$$
\begin{cases}0,333 \leq \eta<0,333 & \text { large gap } \\ 0,333 \leq \eta<0,666 & \text { medium gap } \\ 0,666 \leq \eta<1 & \text { smal gap }\end{cases}
$$

The gap size studied in this paper is at the higher end of the medium gap size range $(c=0.38)$, which the large gap range starts at $\mathrm{c}=0.33)$.

Also, two somewhat distinct but interacting lines of theoretical and experimental research activity can be discerned, relating to long and short cylinders. Stuart (1986) affirms that experiments in long cylinders have shown axisymmetric Taylor vortices and wavy Taylor vortices traveling in the azimuthal direction. On the other hand, experiments in short cylinders have shown that the flow contains a secondary flow in the meridional plane due to end effects. The ratio of the cylinder length to the gap, $\Theta$, is defined by Eq. (12).

$$
\Theta=\frac{L}{d}
$$

\section{Experimental Apparatus}

\section{Taylor-Couette Experimental Apparatus}

The inner and outer cylinders of the Taylor-Couette are made of Plexiglas. The inner cylinder was machined to a radius of $R_{i}=3.14$ $\mathrm{cm}$. The outer cylinder nominal inside radius is $R_{O}=8.18 \mathrm{~cm}$; the cylinders height is $L=102 \mathrm{~cm}$; giving an aspect ratio $\Theta=\mathrm{L} / \mathrm{d}=20$ ( $d$ is the gap length), which is large enough to minimize end effects. Top and bottom endplates are stationary relative a fixed cylindrical coordinates.

The room temperature varied among $22{ }^{\circ} \mathrm{C}$ and $25{ }^{\circ} \mathrm{C}$ and the flow temperature was controlled over a given run with a thermocouple, installed through the bottom endplates. It was found that the flow temperature in this position did not change more significant than the room temperature range. However, in the quartz window position, where the laser beam was introduced, the flow experienced some flow temperature increasing. We could not control the flow temperature in this position, but the laser was operated only while filming and the laser power was reduced to a minimum to prevent a considerable localized heating of the fluid. Additionally, it was verified visually that this flow temperature increasing was not sufficiently to cause a strong free convection and affect the local flow structure.

The experimental system is mounted on a vibration-isolation table. Both inner and outer cylinders have a similar driver system, where the rotation rates can be chosen independently and corotating and counter-rotating cases can are possible. Impulsive starts of the DC motors are used to move the cylinders, which shafts are driven by a frequency of 2100 cycles per minutes. The rotation rates of the cylinders are controlled, using switches with five levels, in which the velocity ramps between successive levels are not known, nevertheless, the acceleration rates between successive levels occurs in short time. Besides of the switches to change the rotation rate of the cylinders, bevel gears with 1:1, 5:1, 25:1 or 125:1 gear ratio are used to couple the motor shaft with the cylinder shaft for both out and inner cylinders, allowing to have more rotation rate levels for inner and outer cylinders.

A small He-Ne laser was directed on to small black tape spot on the inner cylinder wall, and the signal was captured by photo diode, so as to allow the rotation frequency to be measured with aid of a digitizing oscilloscope. The estimation of the rotation rate accuracy was evaluated, applying the Rapid Fourier Transform to the digital time series of the voltage, representing the time revolution of the inner cylinder. On the Fourier transform spectral representation was observed a well-defined frequency peak, with a very strait bandwidth, characterizing a small dispersion of the frequency rotation, included in the range of $\pm 4 \%$ for all case studied, even for low rotation frequency, where the oscillation of the rate rotation was larger.

The Taylor number was varied by changing the inner cylinder rotation frequency. In our experiments only the inner cylinder rotated, $\mu=0$ (see Eq. (8)). Since 0,355 microns wavelength is attenuated by Plexiglas, the outer cylinder was fitted at its midpoint with a small quartz window, approximately $1 \mathrm{~cm}$ in diameter, to facilitate tagging, when we carried out the experiments with the PHANTOMM technique. The quartz window fixation on the inner wall of the outer cylinder was performed with caution, in the way that the surface imperfections caused in this apparatus height were minimized. This was checked, using a plate made specifically for calibrating the level of imperfection of the inner wall of the outer cylinder and outer wall of the inner cylinder. This plate was machined with precision, allowing it to fit within the gap space. The 
deviation of the cylinder walls forming the gap, relative to the plate outer wall, was controlled performing aperture measurements between cylinder walls forming the gap and the plate outer wall, using a computational program developing specifically to evaluate these apertures from digitizing images of CCD camera. The maximum deviation found to the walls forming the gap was 0.18 $\mathrm{mm}$, which can not cause significant influence in the flow structure.

\section{Optical Apparatus}

We have performed the flow visualization study using two techniques:

I. In the first method, the technique termed Particle Streak Imaging, PSI, the visualization was performed using a light sheet from a cw argon ion laser beam oriented in the $(\mathrm{r}, \mathrm{z})$ plane in the annular space between the cylinders (the z-direction is along the vertical axis of the cylinders). The laser beam cut radially through the center of the cylinders and was spread into a vertical laser sheet using a set of mirrors, spherical and cylindrical lenses. The light sheet illuminated the flow with 4 watts power, spread into a $3 \mathrm{~mm}$ thickness and a $30 \mathrm{~cm}$ length. The working fluid was a waterglycerol mixture. The volume ratio of water to glycerol was 4.5:1. Assuming a mean temperature of $24{ }^{0} \mathrm{C}$, our best estimate of the mixture kinematics viscosity, based on the tables of the Handbook of Chemistry and Physics, is $v=1.5 \times 10^{-6} \mathrm{~m}^{2} / \mathrm{sec}$. The flow was seeded with neutrally buoyant 100 micron diameter polystyrene micro spheres with the same density as the water-glycerol mixture. Elastic scattering from the spheres was imaged onto a standard CCD video camera.

II. In the second method, time lines were written into the flow using caged dye photo-activated fluorophore tracers, as described by Lempert, et al. (1995). The technique, termed PHANTOMM, yields quantitative velocity profiles in a manner similar to Laser-Induced Photochemical Anemometry (LIPA) (Falco \& Nocera 1993). Dextran carboxy fluorescein was used as the tracer $(0.5 \mathrm{mgm} / \mathrm{liter}$ in water). In the optical configuration used in this study, the tagging was performed using the third harmonic of a Q-switched Nd:YAG laser at 0.355 micron. Single-pulse energies between $40 \mathrm{~mJ}$ and 60 $\mathrm{mJ}$ were used, depending upon the experiment. Lines were tagged along the radial axis (the r-direction), where the UV tagging beam from the laser was loosely focused with a $30 \mathrm{~cm}$ focal length lens, resulting in a beam waist of approximately $1 \mathrm{~mm}$. The interrogation was performed with a flashlamp pumped dye laser using LD490 dye in methanol with no interactive line-narrowing optics. The dye laser is capable of pulse outputs between $50-400 \mathrm{~mJ}$ with a pulse duration of approximately 2 microseconds. The interrogation beam was formed into a sheet that, for the lateral recording, emerged from the top of the experimental apparatus. The sheet, approximately $2 \mathrm{~cm}$ thick, was formed by mirrors and cylindrical lenses with positive and negative focal length. This sheet was located in the $(r, z)$ plane in the annular space between the cylinders, where the $z$ - direction is along the cylinders axis. For recording from the top view, similar optics were used to place the sheet in the $(r, \theta)$ plane. It covered the entire gap and had also a thickness of approximately $2 \mathrm{~cm}$. A colored glass filter (OG530) was used to block stray elastic scattering from reaching the camera. Fluorescence was imaged onto a standard CCD camera and recorded on a VHS VCR. The delay between the tagging and interrogation lasers was adjusted with a digital delay generator. The maximum repetition rate was $10 \mathrm{~Hz}$.

For both study the flow pattern was imaged using a CCD camera with a image capture rate of 300 images per minute. This CCD camera temporal resolution used, associated with a good enough spatial resolution (defined by the use of lens with large aperture angle, covering all light sheet on the gap area, which was in the worst cases about $9.2 \times 7.6 \mathrm{~cm}^{2}$ ), was sufficient to evince the smaller coherent structure present in the flow. Besides, with aim to guarantee the repeatability of the visualization of the flow structure, was monitored the flow behavior in each Taylor number before acquisission of the images in each experimental run, observing for a long time (fifteen minutes) the images of the flow feature on the image monitor. Thus, it is possible to affirm that the presented results were repeatable.

\section{Results}

\section{Qualitative Analysis}

Results were obtained over a range of Taylor numbers from $0.21 \times 10^{5}$ to $1.1 \times 10^{11}$. In this section are presented photographs oriented in the $(\mathrm{r}, \mathrm{z})$ and $(\mathrm{r}, \theta)$ planes. For both views, the stationary outer cylinder wall appears on the left-hand side of the photographs and the rotating inner cylinder wall appears on the right-hand side. In general, both outer and inner cylinder walls are shown. An exception is Fig. 5 in which a portion of the inner cylinder is shown with a part of the inner cylinder wall.

The visualization using Particle Streak Imaging, PSI, has shown interesting characteristics of the flow from moderate to high Taylor numbers, but it was less revealing for small Taylor numbers. The PHANTOMM technique, however, was more useful at small to moderate Taylor numbers. The combination of the two techniques permitted information to be obtained over a very wide dynamic range.

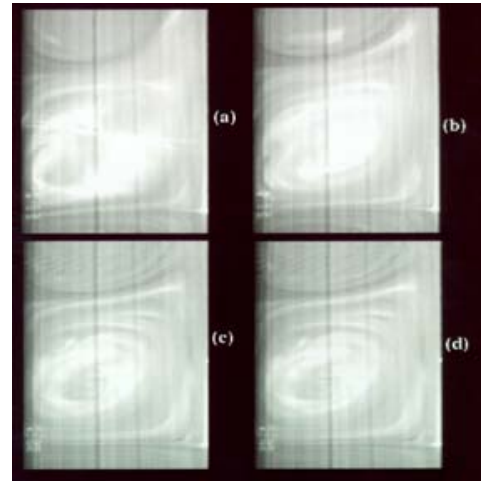

(a)

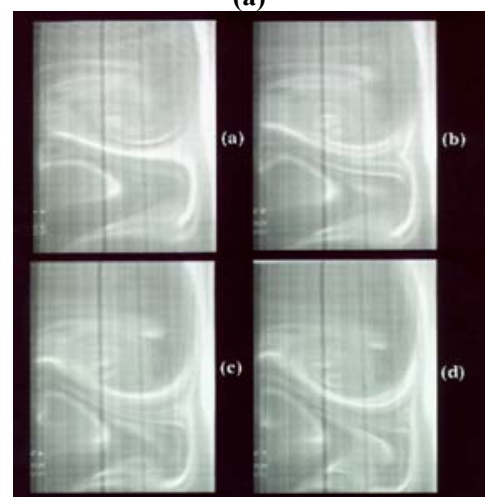

(b)

Figure 1. Collage of representative PHANTOMM images: (a) for Ta=0.21 $\times 10^{5}$ $(\operatorname{Re}=150)$, in the $(r, z)$ plane. Six seconds of delay between successive pictures; e (b) for $\mathrm{Ta}=6.02 \times 10^{6}(\mathrm{Re}=2600)$, in the $(r, z)$ plane. Six seconds of delay between successive pictures. 


\section{Visualization with PHANTOMM Technique}

Figs. 1 to 6 illustrate the use of the PHANTOMM method to elucidate some aspects of the flow structure. The general characteristics of the Taylor-Couette flow for small Taylor numbers are well-defined larger counter-rotating vortices. These cells are typical of the first kind instability (Taylor cells). In each cell, a rotational laminar flow with a high degree of coherence is observed. Fig. (1.a) shows the structure for the smallest rotational velocity our apparatus could achieve $\left(\mathrm{Ta}=0.21 \times 10^{5}\right.$ and $\left.\mathrm{Re}=154\right)$. Taylor cells exhibit a very a slow counter-rotating motion that forms unsteady vortex pairs with their axes in the circumferential direction.

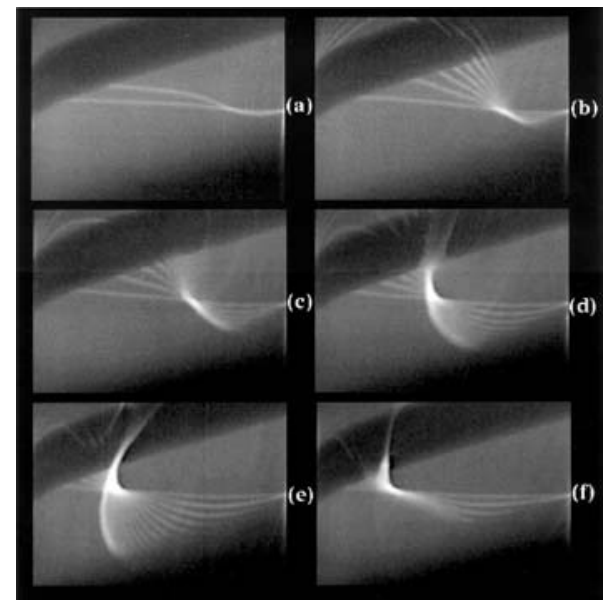

(a)

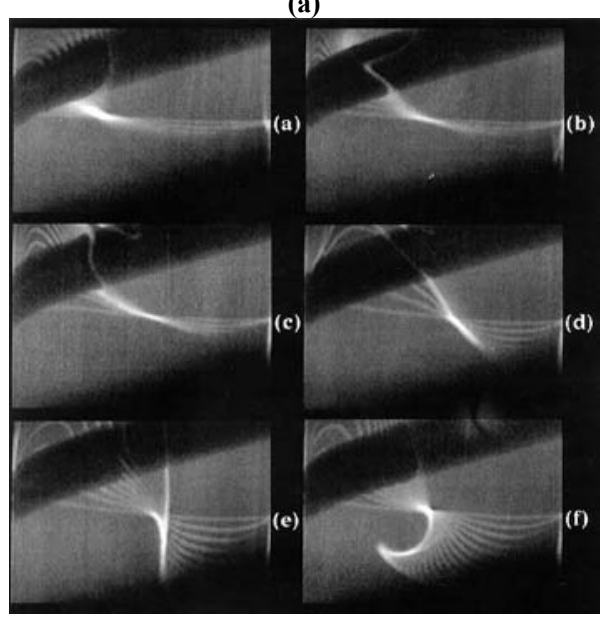

(b)

Figure 2. Collage of representative PHANTOMM line images. Six seconds of delay between successive pictures: $(a)$ for $\mathrm{Ta}=6.02 \times 10^{6}(\mathrm{Re}=2600)$, in the $(r, z)$ plane.; and $(b)$ for $T a=0.21 \times 10^{5}(R e=150)$, in the $(r, z)$ plane.

In the same way, Fig. (1.b) shows the flow structure for $\mathrm{Ta}=6.02 \times 10^{6}$. In this case, Taylor Cells became more regular than those illustrated in the previous figure. In the Figs. (1.a) and (1.b) no turbulence is seen, and the central flow in the gap is dominated by regular cells, equally spaced along the cylinder axis. Cells measure about $6.3 \mathrm{~cm}$ length and have a width spanning the entire gap $(5.04 \mathrm{~cm})$.

Figs. (2.a) and (2.b) illustrate very interesting flow features, whose facts shon with these line images constitute a new flow characteristic in Taylor-Couette. These figures evince the characteristic of flow rotational slow motion within the Taylor cell flow (there is one second of delay between successive photographs). As enhanced in the Figures (2.a) and (2.b), the flow motion in each Taylor cell is characterized by an inflectional point that point outer a shift in the velocity sign axis. This inflectional point constitutes an equilibrium center that moves in the radial direction along the gap, when the time flows. This inflectional point motion can be observed clearly in the photographs of the Figure (2.b) (for example, the inflectional point position is closer to the inner cylinder wall on the third image than the one shown in the fifth image, which represent the flow aspect two seconds later). In the case shown by the Figure (2.b), the inflectional point dislocates in direction of the outer cylinder wall, when it overtakes the gap central line, the axial profile velocity slope changes its sign. In the moment the inflectional point approaches of the out cylinder wall, the rotational sense of the Taylor cell changes and the displacement sense of the inflectional point changes too. This flow feature was verified for all Taylor numbers in the range that well-defined Taylor cells were shown in this visualization study. However, it could be better observed on frame sequences of video records.

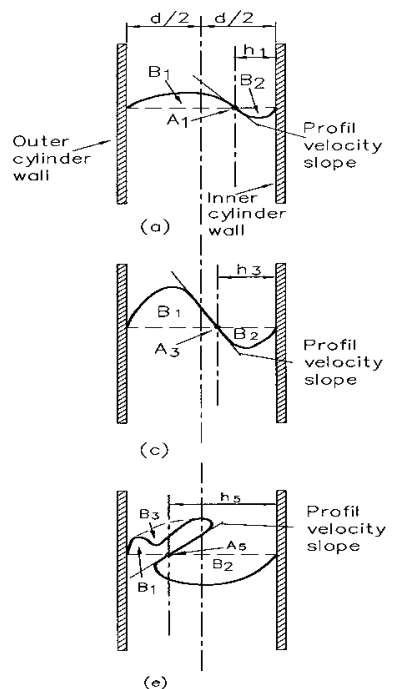

(e)

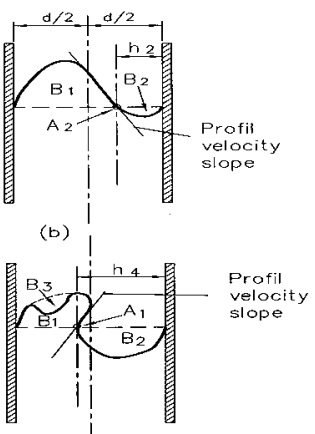

(d)

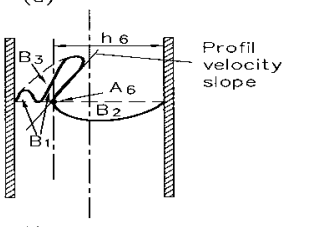

Figure 3. Tracing lines of the flow structure showed on the Figure (2.b).

To assist the clear interpretation of the flow characteristic shown on the photographs presented on the Figures (2.a) and (2.b), it is appealed to the use of the visual images with line tracings of the profile velocities, as shown on the Fig. (3). Similar to the Figures (2.a) and (2.b), in the Fig. (3), the stationary outer cylinder appears on the left-hand side of the figures. The rotating inner cylinder appears on the right-hand side of the figures. The parameters used in Figure 3 to assist the flow feature interpretation are the following: $d$ is the gap space, $h_{i}, i=1,2, \ldots, 6$ represent the distance of the inflectional point of the inner cylinder wall, $A_{i}, i=1,2, \ldots, 6$ represent the inflectional points, $\mathrm{B}_{1}$ shows a zone, where the flow velocity profile present positive velocities, $B_{2}$ and $B_{3}$ show zones, where the flow velocity profile presents negative velocities and the solid lines drawn over the inflectional points represent the profile velocity slopes on the inflectional points. One observes in the Figures (3.a) to (3.c) that the inflectional points are placed closer to the inner cylinder wall than to the outer cylinder wall, where their distances can be represented as $\mathrm{h}_{1}<\mathrm{h}_{2}<\mathrm{h}_{3}<\mathrm{d} / 2$ (where $\mathrm{d} / 2$ 
characterizes the position of the gap central line). For these cases, as shown on the Figures $5 \mathrm{a}$ to $5 \mathrm{c}$, the profile velocity slopes on the inflectional points, represented by $A_{1}, A_{2}$ and $A_{3}$, have negative signs. On the other hand, one can also remark on the Figures $5 \mathrm{~d}$ to $5 \mathrm{f}$ that the inflectional points overtake the gap central axial line and they are placed closer the out cylinder wall than the inner cylinder wall, where their distances can be represented as $\mathrm{d} / 2<\mathrm{h} 4<\mathrm{h}_{5}<\mathrm{h}_{6}$. In these cases, the profile velocity slopes on the inflectional points, represented by $\mathrm{A}_{4}, \mathrm{~A}_{5}$ and $\mathrm{A}_{6}$, have the positive signs. One observes also that when the velocity profile slope changes its sign, the flow within the Taylor cell becomes unstable and appears within the zone of positive velocities, $B_{1}$, a zone with negative velocities, $B_{3}$. When the velocity profile inflectional point approaches more to the outer cylinder wall, after it overtakes the gap central line, the velocity axial profile trend in the zones $B_{1}$ and $B_{3}$ are that the zone $B_{1}$ disappears and the zone $B_{3}$ occupies all the region. Thus, the zone $B_{3}$ will represent the velocity profile between the inflectional point and the outer cylinder wall, since at this time occurs the inversion in the flow velocity sense within the Taylor cell. In the same way, the zone $\mathrm{B}_{2}$ that represents a negative profile velocity in the region between the inflectional point and the inner cylinder wall shrinks its magnitude up to a complete inversion in the flow velocity sense in this region. At this time occurs the inversion in the flow motion sense within the Taylor Cell and a similar mechanism happens when the inflectional point now displaces in the counter sense. This mechanism that characterizes the Taylor cells behavior in the $(r, z)$ plane has a behavior similar to a pendular cycle motion.

This pendular cycle motion can be clearly evidenced observing the videotape in real-time. Figure (2.a) shows the pendular cycle motion in the sense inverse of the one shown on the Figure (2.b). In the case shown in the Fig. (2.b), the inflectional point displaces from the outer cylinder wall to the inner cylinder wall. The instability attained by the inflectional point, in this case, was not the one of the slope sign inversion. At first, as shown in Fig. (3.e), the inflectional point slope becomes infinite, and in the second step, as shown in the Figure (3.f), the flow assumes a complex configuration close to the inflectional point, with high degree of instability. There, in a round region, the flow is swept out in azimuthal direction and immediately, the cells begin the inversion in the sense of the pendular cycle motion. This complex flow feature could be understood better with the helpful aid of the real-time videotape observation.

The flow aspect described is clearly enhanced by images in Fig. (2.a), where the inflectional point moves from close to inner cylinder wall to outer cylinder wall. In the same way, in Fig. (2.b), the inflectional point moves from close to outer cylinder wall to inner cylinder wall, characterizing pendular cycle motion. However, for higher Taylor numbers, the inflectional point never overtakes the gap central line, located between the central line and the inner cylinder wall, evincing that the motion of the inflexional point along the gap in the radial direction is restrained when the Taylor number increases.

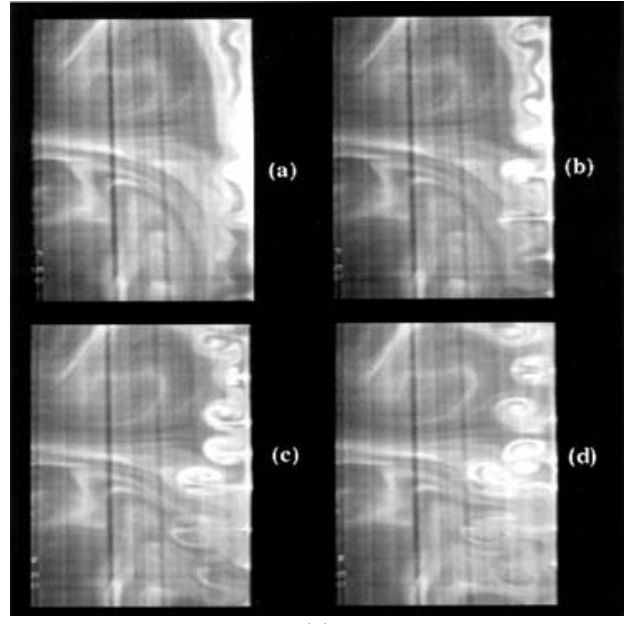

(a)

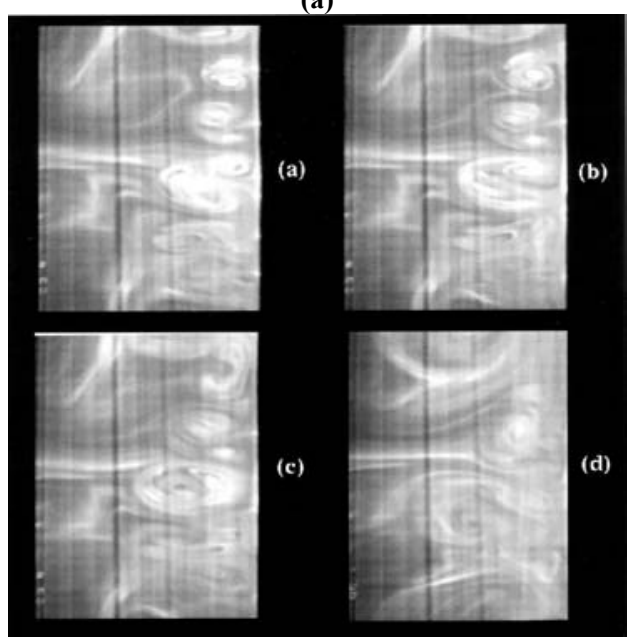

(b)

Figure 4. Collage of representative PHANTOMM images. One second of delay between successive pictures. (a) for $\mathrm{Ta}=6.02 \times 10^{6}(\operatorname{Re}=2600)$, in the $(r, z)$ plane; and $(b)$ for $T a=6.02 \times 10^{6}(\operatorname{Re}=2600)$, in the $(r, z)$ plane.

By contrast, when the Taylor numbers increase, the flow pattern in the gap central region contains similar cells, but with a more irregular appearance. The flow is intermittently turbulent. The flow inside the Taylor cell displays the onset of another instability by the presence of smaller counter-rotating vortex pairs. These counterrotating vortices are called the Görtler vortices, vortex pairs or secondary instability. The vortex pairs, which appear in the flow for low Taylor number, are small and develop inside the Taylor cells and close to the inner cylinder wall. For larger Taylor numbers, the flow inside the Taylor cells becomes more turbulent and the Görtler vortex pairs occur unsteadily and become very large. Sometimes these vortices appeared inside the Taylor cells or close to the inner cylinder wall, but more commonly they occurred in the shear layer between the Taylor cells. These vortex pairs appear constantly in the flow and they play an important role in the transition process to turbulence. Figs. (4.a) and (4.b) illustrate these flow aspects.

Figs. (5.a) and (5.b) show the representative PHANTOMM line images. The images in the $(r, \theta)$ plane, for the lower value of the Taylor number, at $\mathrm{Ta}=0.21 \times 10^{5}$, has been observed the threedimensional characteristics of the flow. 


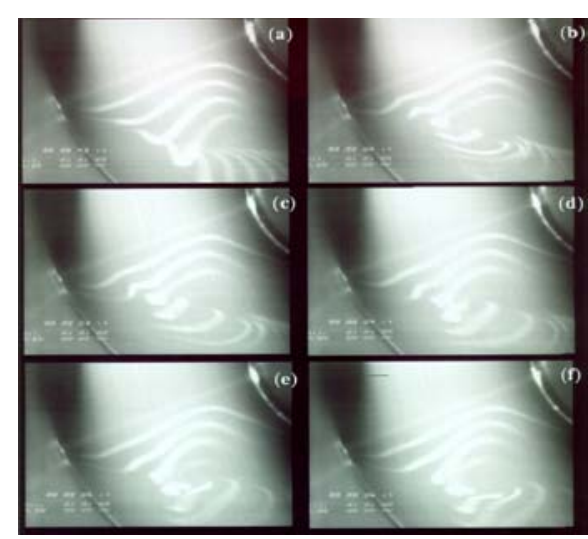

(a)

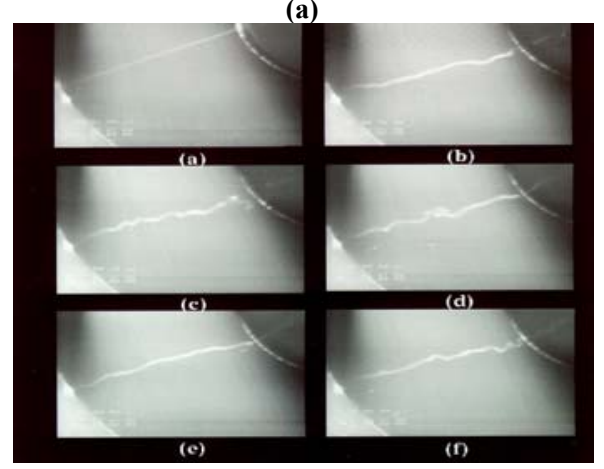

(b)

Figure 5. (a) Collage of representative PHANTOMM line images for $T a=2.3 \times 10^{6} \quad(\operatorname{Re}=1600)$, in the $(r, \theta)$ plane (the images from (a) to (f) correspond to the interrogation lines with one second of delay between successive lines. And two seconds of delay occur between successive pictures; and (b) Collage of representative PHANTOMM line images for $\mathrm{Ta}=2.45 \times 10^{8}\left(\operatorname{Re}=1.66 \times 10^{4}\right)$, in the $(r, \theta)$ plane $((a)$ baseline image with $d t=1$ $\mathrm{ms}$ and the images from (b) to (f) correspond to the interrogation lines with $d t=1 \mathrm{~ms}$ and one second of delay between successive pictures).

The lines close to the outer cylinder wall are affected by the axial flow in the vertical direction. The gap core region has a nozzle appearance due the flow interaction in the $(r, \theta)$ and $(r, z)$ planes. The flow structure indicates a larger velocity in the gap central region than that for the inner cylinder wall because of the nozzle effect. A clearer three-dimensional characteristic is shown in the Fig. (5.a), which represents the images in the $(\mathrm{r}, \theta)$ plane, for $\mathrm{Ta}=2.3 \times 10^{6}$. It illustrates the flow behavior inside the Taylor cells, where line images are strongly twisted. When Taylor number increases the regular flow feature changes and the Taylor cells coherence shrinks. At the same time, fluctuations with higher frequencies appear in the flow (Fig. (5.b)). In the same way, PHANTOMM line images in the $(r, z)$ plane show the same remarks presented above, analysing the images in the $(r, \theta)$ plane.

Flow Structure Visualization with Particle Streak Imaging, PSI

Figs. (6.a) and (6.b) illustrate the flow visualization with Particle Streak Imaging, PSI, which characterizes the structure evolution over a range of Taylor numbers from $2.7 \times 10^{5}$ to $6.8 \mathrm{x}$ $10^{10}$

It was observed the presence of Taylor cells at $\mathrm{Ta}=2.68 \times 10^{5}$. This Taylor number is the smallest number in which Taylor cells were observed in this study with Particle Streak Imaging, PSI. The critical Taylor numbers published in the literature for medium gaps are significantly smaller than these and are approximately equal to that corresponding to Fig. (1.a). It should be noted that, however, almost all published critical Taylor numbers for both medium and large gaps were found by interpolating analytical solutions, and not by direct experiment. For example, the critical Taylor number $(\mathrm{Ta}=2,337)$ value given by Smith and Townsend (1982) was obtained by an interpolation from the analysis by Robert (1965). In the same way, Wei et al. (1992) found the critical Taylor number, for both medium and large gaps $\left(\mathrm{Ta}=3,120\right.$ and $\mathrm{Ta}=0.44 \times 10^{5}$, respectively), by interpolation between the analytical values calculated by Sparrow et al. (1964) and Walowit et al. (1964). We believe that the critical Taylor number for our flow found using the PHANTOMM Technique is probably less than $\mathrm{Ta}=0.21 \times 10^{5}$, but this could not be verified.

Wei et al. (1992) studied Taylor-Couette Flow in both medium and large gaps. In their study of a large gap $(c ̧=0.084)$ they covered Taylor numbers in the range $1.55 \times 10^{6}$ to $4.64 \times 10^{7}$. Taylor cells that spanned the entire gap were never observed. However, in their study with a medium gap $(\eta=0,500)$, they noted that Taylor cells were present in the flow, but it may be possible that what they called a Taylor cell is what we call a Görtler vortex that is an instability confined to a thin layer on the inner wall.

In our experiment, small Görtler vortex pairs were observed inside the Taylor cells and near the inner cylinder wall when the Taylor number increases. The smaller vortices move quickly inside the cells and the flow appears to be more turbulent. The Taylor cells size changes constantly in the flow, and they oscillate in the direction of the cylinder axis. Nonetheless, one can clearly distinguish the Taylor cell (observing the videotape in real-time illustrates these aspects more clearly).

Indeed, the explanation to be presented along the text related with the PSI technique figures evinces an important purpose of the research. It was intended along the flow visualization description to show the influence of the large scales, as the Taylor cells, on the turbulence transition in a large Taylor number range; and to enhance also, if there is the presence of Taylor cells in high turbulent flow, how they force the behavior of the turbulence and how it may be linked to the system geometry. Nevertheless, although the flow visualization presentation evinced, almost clearly, these facts, it can be improved if are used figures with tracing lines of the flow structure similar to the ones shown in Fig. (6.b). The tracing lines of the flow structure presented on Fig. (6.b) have the aim to aid in the flow visualization description for high Taylor numbers, at this point the flow configuration became complex and irregular, thus it was difficult to enhance it with a limited set of photographs, in spite of the flow configuration described in the text for high Taylor numbers is clear when observing the videotape in real-time.

When the Taylor number increases, cells become more turbulent. Even at quite high Taylor numbers the flow is dominated by large Taylor cells $\left(\mathrm{Ta}=1.47 \times 10^{10}\right)$. However, cells become more irregular and the flow inside the cells become more turbulent when the Taylor number increases, and although the outflow (flow in the gap core) presents high degree of turbulence, the Taylor cells are still identifiable. For higher Taylor number, the disturbance in the flow increases, and as a consequence the flow becomes more homogeneous inside the Taylor cells, making it more difficult to identify the cells. The Taylor cells appear to break up, and for even larger Taylor numbers the homogenization occurs due to the turbulence spreads across the gap and the flow structure changes its patterns. At $\mathrm{Ta}=2.02 \times 10^{10}$, a toroidal vortex in helical motion develops in a thin layer on the inner cylinder wall (see bright spot on the inner cylinder on Figure (6.a) that can be seen clearly on videotape in real-time). One can suggest that this helical toroidal 
vortex constitutes instability of the third kind. While the quality of the flow visualization, at this point up to higher Taylor numbers was not good enough to clearly observe the flow structure, so to explain better the flow behavior, it is used to assist the interpretation of the visual images, tracing lines of the photographs that are included in Fig. (6.b).

As shown in the photograph of Figure (6.a), a similar flow pattern is presented in Figure (6.b). In this figure the regions shown by $B_{1}$ represent the Taylor cells and the helical toroidal vortex is represented as lines involving the inner cylinder wall.

An other important fact observed related with the helical toroidal vortex is that it concentrates more intensely in the wall of the inner cylinder located in the levels of intersections between the Taylor cells, as shown in Figure (6.b), whose positions of the largest toroidal vortex concentrations are represented by $h_{1}$ and $h_{2}$. In the same way, this flow characteristic is evinced clearly on the photograph in Figure (6.a), in which time bright spot on the inner cylinder wall represents the helical toroidal vortex. It can also be observed that the positions of the largest helical toroidal vortex concentrations move up and down along the axial direction of the inner cylinder, as a function of the Taylor cell motions in this direction.

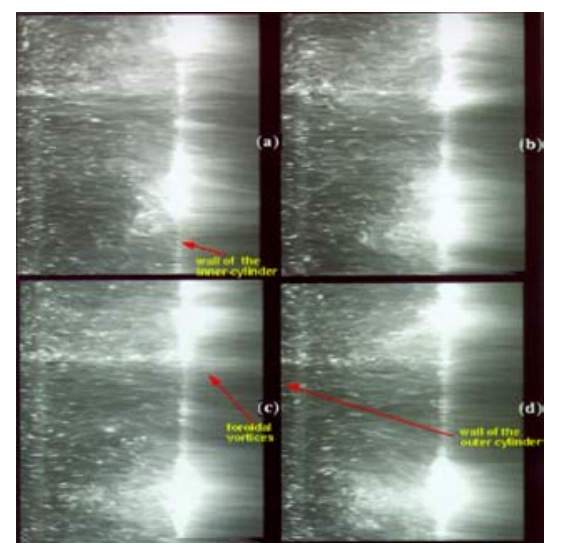

(a)
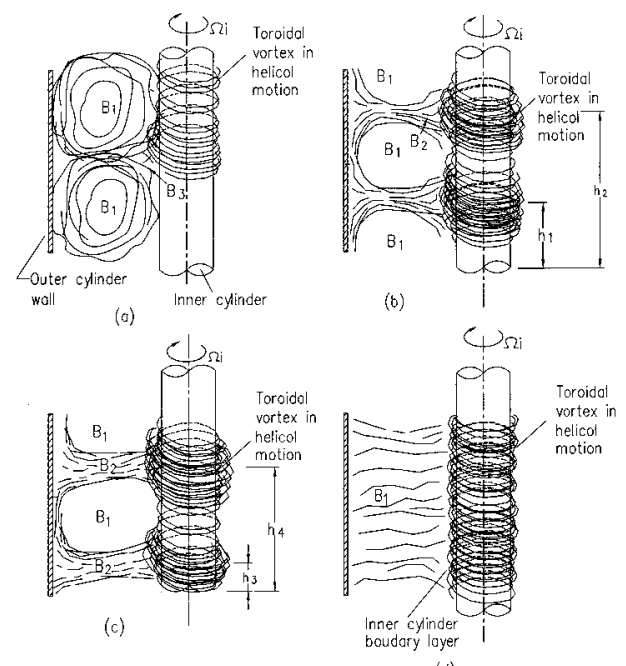

(d)

(b)

Figure 6. (a) Flow structure visualization with PSI, for $\mathrm{Ta}=3.7 \times 10^{10} \cdot 0.133$ seconds of delay between successive pictures; and (b) Tracing lines of the flow structure showed on the Fig. (6.a).
Thus, the images $b$ and $c$ in Figures (6.b) enhance this flow pattern, where $\mathrm{h}_{3}<\mathrm{h}_{1}$ and $\mathrm{h}_{4}<\mathrm{h}_{2}$, characterizing the moving down of the position of the largest helical toroidal vortex concentrations. This axial motion of the largest helical toroidal vortex concentrations is also shown clearly on the image $a$ and $d$ in Figures (6.a). At higher Taylor numbers, this helical toroidal vortex covers the entire inner cylinder wall and forms over its wall a thin layer with an irregular appearance, which is characterized by an irregular concentration of the helical toroidal vortex along the inner cylinder wall (Fig. (6.a)).

There is a strong evidence that vortex pairs lead the flow transition to turbulence, therefore they have been subject of studies in several previous experiments. In particular, Wei et al. ${ }^{4}$ observed the presence of Görtler vortex pairs from moderate to high Taylor number for medium gap $\left(1.99 \times 10^{6}<\mathrm{Ta}<9.1 \times 10^{7}\right)$. In their description, the Görtler vortex pairs form in the boundary layer close to the inner cylinder wall and then they are advected by the outflow. Also, they noted that the presence of Görtler vortex pairs is an exception in some cases. These cases included the low Taylor number for all three gap sizes that they studied and high Taylor number for the small gap. With a small gap, counter-rotating vortex pairs were observed to form very infrequently at the inner cylinder wall. Smith and Townsend (1992) found that, for small Taylor numbers (less than $4.7 \times 10^{8}$ ), radial velocity shows almost perfectly periodic fluctuations, but breaks and phase jumps become noticeable for Taylor number over $9.4 \times 10^{8}$. In this case, periodicity was still observed, but the wave forms were far from sinusoidal, being peaked but symmetrical around the flow center, and asymmetric near the flow boundaries. The oscillations coherence degree may be assessed more precisely from measurements of autocorrelations. Measurements by these authors had a local character, and we suggest that their description was related with the behavior of the Görtler vortex pairs. This explanation suggests that the appearance of Görtler vortex pairs presage the transition to turbulence in Taylor-Couette flow, and our visualization study supports this conclusion.

Our study demonstrated that interplay between small and large scales present in the flow and showed the gradual transition to turbulence in the flow at high Taylor numbers. Taylor cells were found over a large range of Taylor numbers $(0.21 \times 105<\mathrm{Ta}<6.81$ $\mathrm{x} 10^{10}$ ) until highly turbulent flow appeared. At low Taylor numbers, the flow in the cells appears to be a rotational laminar flow with a high degree of coherence. When the Taylor number is increased, the aspect of the cells becomes more irregular, and the flow inside the cells becomes turbulent. These cells have a strong imprint on the turbulence in Taylor-Couette flow that is linked with the system geometry and a specific time scale. We have provided supporting evidence for previous suggestions that the flow transition from laminar to turbulent is led by the generation of Görtler vortices, which develop inside the Taylor cells close to the inner cylinder wall. The presence of Taylor cells even in highly turbulent flow suggests that the turbulence mechanism in this flow is constrained by these cells. For example, the Taylor cells influence the location of the Görtler instability, its geometrical form, and its oscillation in time. This observation appears to be new.

Disturbances in the flow grow as Taylor numbers increase. At high Taylor numbers, the flow presents more homogeneous turbulence close to the inner cylinder wall and in a large part of the outer flow. Eventually, Taylor cells break down. At Ta $=1.47 \mathrm{x}$ $10^{10}$, the turbulence homogenization has spread across the entire gap and the flow structure has changed its nature. A toroidal vortex in helical motion develops in a thin layer on the inner cylinder wall. This flow pattern is similar to that of boundary layer flow in 
concave surfaces. This transition has not, to our knowledge, been noted in previous Taylor-Couette flow studies.

\section{Quantitative Results}

We have applied the PHANTOMM technique to measure the instantaneous velocities in the $(r, z)$ and $(r, \theta)$ planes. Images from both views were recorded at 40 different Taylor numbers in the range $0.21 \times 10^{5}<\mathrm{Ta}<0.11 \times 10^{12}$ and for several different delays between tagging and interrogation.

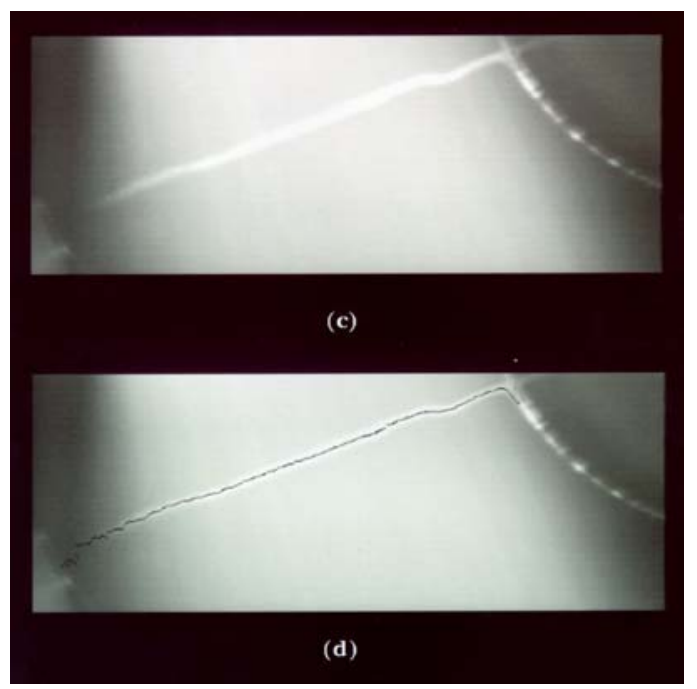

Figure 7. (c) typical view of the interrogation line in the $(r, \theta)$ plane. $15 \mathrm{~ms}$ after tagging for $\mathrm{Ta}=5.96 \times 10^{7}(\mathrm{Re}=8176)$ and $(d)$ the corresponding fitted line position.

Fig. (7) shows a typical interrogation line, overlaid with an estimate of the line center estimated using the gray scale intensity as a function of $r$. The center of intensity was found using a peak searching algorithm. For the measurements in the $(r, z)$ plane, the algorithm simply located the pixel corresponding to the maximum gray scale intensity over each vertical "slice" of data. For the measurements in the $(r, \theta)$ plane, the center of intensity was found in two steps: as a first approximation, the maximum intensity pixel in a vertical "slice" was located, as above for the $(r, z)$ plane. Since the principal flow direction is along theta, a set of 30-40 pixels about each "vertical" maximum, normal to the radial direction, was, subsequently, evaluated. The maximum intensity pixel of each of these sets was taken as the interrogated line center point as a function of $r$.

The calibration of the image magnification was performed externaly to the experimental system because of the difficulty in performing this in-situ. We utilized an image of a grid placed in the same optical geometry used for the experiments. In this calibration, we did not apply corrections for the distortion caused by viewing the tagged lines through the cylindrical surface in the $(r, z)$ plane. Also, we did not correct the distortion caused by the mismatch of the indices-of-refraction for air and water. However, by comparing the known gap length to that, determined using the calibration measurements, we estimated the error to be less than $3 \%$. The principal effect of the cylindrical surface is to compress the radial scale of the image very near the outer wall. The z-axis is not affected, except for a very small translation. This translation was minimized by positioning the camera at the same height as the tagging laser. A conservative estimate of the absolute velocity uncertainty introduced by these effects is less than $\pm 5 \%$.

Figures 8 to 11 show the dimensionless instantaneous velocities across the gap for the planes $(r, \theta)$ and $(r, z)$ for several Taylor numbers in the range of $0.21 \times 10^{5}<\mathrm{Ta}<0.11 \times 10^{12}$. In each figure. Curves were put on the same scale in order to illustrate relative fluctuations in the two planes as a function of Taylor number. In all situations, the flow exhibits large spatial fluctuations.

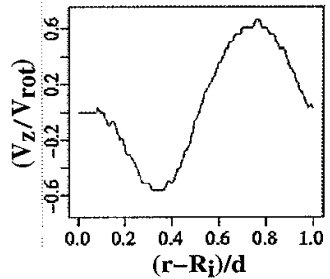

(a)

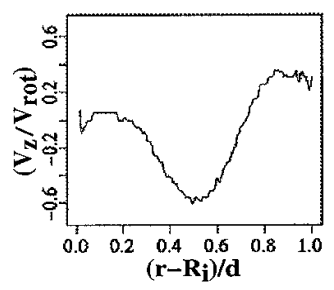

(c)

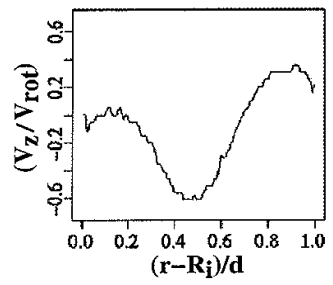

(b)

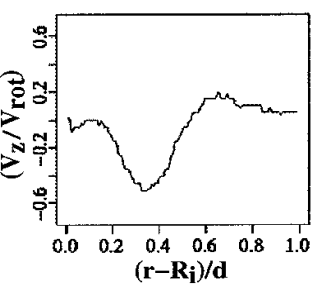

(d)
Figure 8. Dimensionless instantaneous velocities along the gap, in the plane $(r, z)$, for $\mathrm{Ta}=0.21 \times 10^{5}$, Sixteen seconds of delay from (a) to (d).

Velocity profiles shown in Figs.(8) and (9) correspond to the $(r, z)$ plane measurements. One can observe in the Fig. (9) that the velocity profile is characteristic of a laminar rotational flow, typical of Taylor cells with a very slow circulation velocity. When the Taylor number increases, the characteristic of laminar rotational flow disappears and is replaced by flow with larger fluctuation with irregular aspects. At all Taylor numbers, the mean velocity is zero in $(\mathrm{r}, \mathrm{z})$ plane.

Figs. (10) and (11) show the velocity profiles for several Taylor numbers in the $(\mathrm{r}, \theta)$ plane. In Fig. (10), which corresponds to $\mathrm{Ta}=$ $0.21 \times 10^{5}(\operatorname{Re}=154)$, we observe that the velocity in the gap center achieves an amount two times greater than the linear velocity of the inner cylinder wall. This occurs because of the three-dimensionality and the resulting streamwise convergence, as indicated earlier. The mean velocity in the $(r, \theta)$ plane becomes less affected by the threedimensional characteristic of the flow as the Taylor number increases. Moreover, the outflow velocity profile evolves with the Taylor number, and it achieves an almost constant slope (about 0.42 ) when the Taylor number becomes larger than $5.96 \times 10^{7}$. 


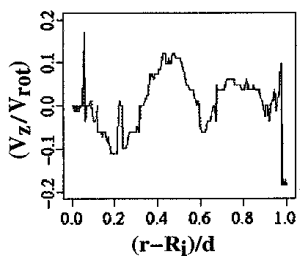

(a)

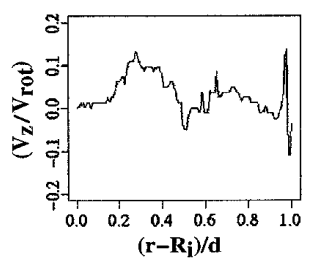

(c)

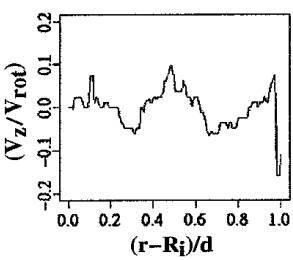

(b)

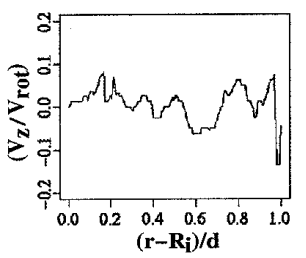

(d)
Figure 9. Dimensionless instantaneous velocities along the gap, in the plane $(r, z)$, for $T a=0,48 \times 10^{11}$, Four seconds of delay from (a) to (d).

One can see this tendency in the velocity profiles of Fig. (11). This behavior permits the enhancement that the outflow velocity profile is led for an universal profile in a high Taylor number. In the same way, the outflow means velocity in the $(\mathrm{r}, \mathrm{z})$ plane evolves with the Taylor numbers and it achieves a constant slope about zero (Fig. (9)).

Almost all variation in the angular momentum occurs within the two wall boundary layers since surface stresses are expected to be nearly proportional to the $7 / 5$ power of the rotational velocity, according to Smith and Townsend (1982). One can observe that the boundary layer in the inner cylinder wall is concentrated in a region within 0.07 of the gap length. The thickness of the boundary layer shrinks when the Taylor number increases. For larger Taylor numbers, this thickness is less than 0.03 of the gap length. This boundary layer thickness on the outer cylinder wall is larger than that of the inner cylinder wall. However, the flow in this outer boundary layer appears to be more disturbed, fluctuating more intensively close to the wall. This fact suggests that flow in the boundary layer on the outer cylinder is more affected by curvature. It must be repeated, however, that optical distortions are maximum near the outer wall, and it is, therefore, difficult to quantify the boundary layer thickness.

We have evaluated the angular-momentum ratio defined by the following equation:

$$
\left(\frac{\Gamma}{\Gamma_{\text {rot }}}\right)=\left(\frac{\mathrm{V}_{\mathrm{i}} \mathrm{r}}{\mathrm{V}_{\text {rot }} \mathrm{R}_{\mathrm{i}}}\right) \text { with } \quad \mathrm{i}=\theta \text { or } \mathrm{z}
$$

where, $\mathrm{V}_{\mathrm{i}}$ is the velocity in the $\theta$ and $\mathrm{z}$ direction, $\mathrm{V}_{\text {rot }}$ is the rotational velocity and $R_{i}$ is the radius of the inner cylinder.

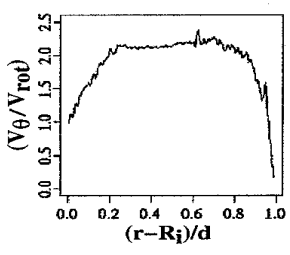

(a)

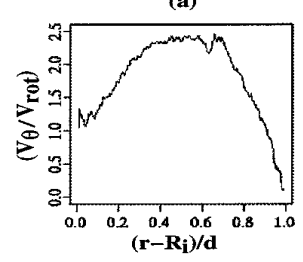

(c)

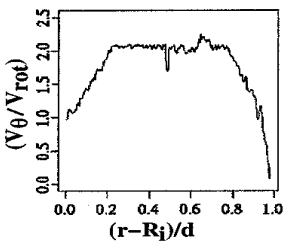

(b)

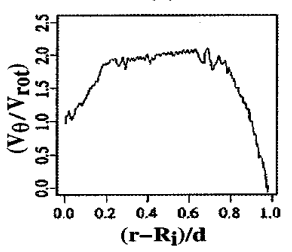

(d)
Figure 10. Dimensionless instantaneous velocities along the gap, in the plane $(r, \theta)$, for $\mathrm{Ta}=0.21 \times 10^{5}$, sixteen seconds of delay from (a) to (d).

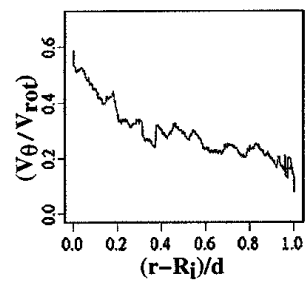

(a)

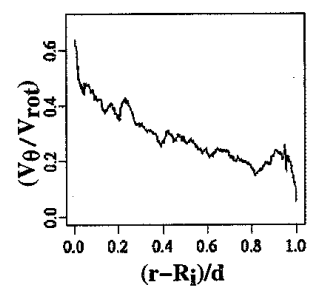

(c)

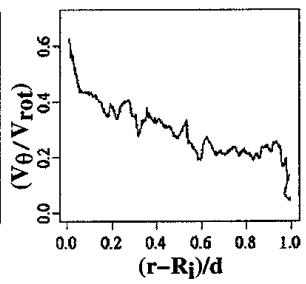

(b)

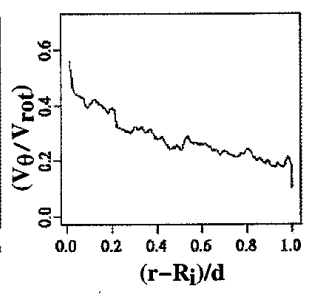

(d)
Figure 11. Dimensionless instantaneous velocities along the gap, in the plane $(r, \theta)$ for $\mathrm{Ta}=0,48 \times 10^{11}$ four seconds of delay from (a) to (d)

The mean angular-momentum is almost constant across the gap for larger Taylor numbers. For the measurements in the $(r, q)$ plane, the mean angular-momentum ratio is close to 0.50 , when the Taylor numbers are larger than $6.02 \times 10^{6}$ (this value is the same found by Smith and Townsend, 1982). This fact suggests that for high Taylor numbers the outflow is not affected by the curvature effect associated with the cylinder surfaces, and for this circumstance it is similar to boundary layer flow in channels. Nevertheless, for the measurements in the $(\mathrm{r}, \mathrm{z})$ plane, the mean angular-momentum ratio is constant and it is zero, even when Taylor numbers are larger than $6.02 \times 10^{6}$. In the same way, the outflow in this plane is not affected by the cylinder surfaces curvature .

From Equation 9, we can obtain one equation to determine the outflow velocity in the plane $(\mathrm{r}, \theta)$, for Taylor numbers larger than $6.02 \times 10^{6}$, which may approximate the outflow velocity in region of $90 \%$ of the gap. This equation is the following:

$$
\mathrm{v}_{\theta}=0.5 \mathrm{v}_{\operatorname{rot}}\left(\frac{\mathrm{R}_{\mathrm{i}}}{\mathrm{r}}\right)
$$


The spatial one-dimensional and one-sided spectral density function gives further information about the flow behavior. For example, the spectral density function of a periodic signal gives a maximum in the characteristic wave number of the signal. When the dynamic system has a quasi-periodic behavior, then the spectral density function presents several maxima that correspond to the wave numbers of unrelated phenomena. On the other hand, the spectral density function for a random signal has a continuous spectral representation over a large bandwidth of wave numbers that characterizes a disordered behavior. The disorder degree of a system is associated with the wave number bandwidth of the spectral representation.

The spectral density function was determined for several Taylor numbers in the range $0.21 \times 10^{5}<\mathrm{Ta}<0.11 \times 10^{12}$ (Biage et al., 1996). Physically, these figures represent the energy flow as a function of the wave number. The flow feature characterized by these results is typically toroidal eddies with a regular wave number $(\mathrm{K}=0.198 \mathrm{~cm}$ $\left.{ }^{1}\right)$, that is, the Taylor cells, which have a characteristic scale with the same dimension of the gap (see also the flow visualization presented in Biage et al. (1996)). The spectral density function represents other maxima with significant energy (amplitude) when the Taylor number increases. In reality, the flow becomes more irregular, with important contributions to characteristic scales less than the fundamental scale (approximately 0.198). The flow aspect shown by these results is almost perfectly periodic, for Taylor numbers less than $5.96 \times 10^{7}$. For Taylor numbers larger than this, the flow structure is quasi-periodic with contributions from several characteristic wavelengths. This flow structure persists for the higher Taylor numbers studied in this paper.

The spectral density function, which physically, represents the flow oscillation energy as a function of the wavenumbers. The flow feature is characterized by toroidal eddies with regular wavenumbers. At low Taylor numbers, the vortices in the flow with predominance energy are toroidal eddies with a wavenumber $\mathrm{K}=0.198 \mathrm{~cm}^{-1}$, that is typically the Taylor cells, which have characteristic scales with the same gap dimension. However, as the Taylor number increases the flow pattern becomes more irregular and the spectral density function present other maxima with significant energy (amplitude), whose wavelengths are multiple scales with a factor two (e. g., high scales with smaller wavelengths have commensurately related wavelengths, where a factor two is the ratio between two successive maxima). In reality, when the flow feature becomes more irregular, it has important energy contributions from the small scales, in which their contributions can be either of the same order or even larger than the one of the fundamental scale, depending of the Taylor number. At high Taylor numbers, the energies of the characteristic scales with small wavelengths exceed the energy of the fundamental scale energy. Thus, the scales with predominant energies in the flow shrink their wavelengths when the Taylor number increases further. Therefore, it was performed the vortex wavelength measurements, taking the maxima found in the digital values of the spectral density function.

Figure (12) is a plot of vortex wavelengths non-dimensional zed by the outer-cylinder radius versus a characteristic Reynolds number squared, powered $-1 / 3$, where the Reynolds number is

$$
\operatorname{Re}_{0}=\left(\frac{\Omega_{\mathrm{i}} \mathrm{R}_{0}^{2}}{\mathrm{v}}\right)^{2},
$$

As prescribed by Barcilon \& Brindley (1984), Fig. (13) is a plot of vortex wavelengths scaled on inner-cylinder circumferential velocity and the kinematics viscosity of water versus the ratio $\left(\mathrm{Ta} / \mathrm{Ta}_{\text {crit }}\right)^{-2 / 5}$.

In Figs. (12) and (13), the solid squares represent the vortex wavelength measurements, as explained, obtained from the computed spectral density function, the open squares represent the vortex wavelengths with maximum energy levels and the open circles with solid line is the fitting curve that was obtained from the vortex wavelengths measurements with maximum energy levels, and finally, in Figure 13 the open up triangle with solid line on the Figure 12 is the analytical result of Barcilon \& Brindley (1984), defined as

$$
\frac{\lambda}{\mathrm{R}_{0}}=31.89\left(\mathrm{Re}_{0}\right)^{-1 / 3}
$$

which were reproduced, using the small-gap approximation and the method of matched asymptotic expansions.

It is observed in Figure 12 that it is remarkable how well the analytical results of Barcilon \& Brindley (1984) agree with the small scales verified in the flow. More precisely, these authors obtained the following relation between Görtler wavelength and their characteristic Reynolds number:

$$
\frac{\lambda}{R_{0}} \approx G_{c}^{1 / 3}\left(R_{0}\right)^{-1 / 3}
$$

where is the Görtler wavelength and $G_{c}$ is the critical Görtler number for the onset of the Görtler vortices.

Thus, considering that the analytical results of Barcilon \& Brindley (1984) were defined to represent the Görtler wavelengths as a function of the $\mathrm{Re}_{0}$, it is recommendable to consider that the smaller coherent scales verified in the vortex wavelength measurements of this study are the Görtler vortices. This argumentation is construed with an additional strong evidence that in this study the Görtler scales were verified in the Reynolds number squared range as $2.4 \cdot 10^{7} \leq\left(\operatorname{Re}_{0}\right)^{2} \leq 8 \cdot 10^{9}$, which is almost the same Reynolds number squared range that Wei et al. (1992) verified the Görtler vortices; e. g., $3 \cdot 10^{7} \leq\left(\mathrm{Re}_{0}\right)^{2} \leq 1 \cdot 10^{9}$. In Wei's research the superior limit of the Reynolds number squared range is lower, however, in that study $\left(\operatorname{Re}_{0}\right)^{2}=1 \cdot 10^{9}$ was the maximum value of the Reynolds number squared analyzed in the experiments.

Other important fact observed in Figure 12 is that the data representing the scales with maximum energy levels do not follow the $-1 / 3$ power dependence, as described by Eq. (15), referent the analytical results of Barcilon \& Brindley (1984). These data are described by the following fitting curve:

$$
\frac{\lambda}{\mathrm{R}_{0}}=2.81 \cdot\left(\operatorname{Re}_{0}^{2}\right)^{-2 / 15}
$$

The behavior of the data corresponding to the scale wavelengths with predominance energies in the flow, which follow the $-2 / 15$ power dependence (Eq. 18), illustrates clearly, in a quantitative way, the strong influence of the large scales on the flow pattern. The scale wavelengths with predominance energies shrink their size when the Taylor number increases (it is proportional to the Reynolds number squared). As described the manuscript along about flow visualization at low Taylor numbers, the regular Taylor cells exhibit a slow counter-rotating motion that forms unsteady vortex pairs with their axes in the circumferential direction, equally spaced along the cylinder axis. By contrast, when the Taylor numbers increase, the 
flow pattern in the gap central region contains cells, but with a more irregular appearance. For larger Taylor numbers, the flow inside the Taylor cells becomes more turbulent and the Görtler vortex pairs occur unsteadily and become very large, as shown in the Figures 5 and 6 in the text. Sometimes these vortices appeared inside the Taylor cells and close to the inner cylinder wall, but more commonly they occurred in the shear layer between the Taylor cells. The Görtler vortices move quickly inside the cells and the flow appears to be more turbulent. The Taylor cells' size change constantly in the flow, and they oscillate in the direction of the cylinder axis. There, the outflow presents a high degree of turbulence, but the Taylor cells are still identifiable. As the Taylor number increases farther, the disturbance in the flow increases, and as a consequence the flow becomes more homogeneous inside the Taylor cells. These cells assume irregular wavelengths due to the strong interaction among the cells and the streaky structure the inner cylinder boundary layer.

The complex flow pattern described in the above paragraph is consistently represented by the data of the coherent scale wavelengths, plotted in Fig. (12) as solid square. As can be observed in Fig. (12), these data involve a set of coherent scale wavelengths in the interval comprised of a spectrum, defined from the large Taylor cells up to the small scales as the Görtler vortices. Clearly, it is consist that the scales with maximum energy levels are defined by larger scales than the Görtler vortices, also, that the coherent scale wavelengths shrink as the Taylor number increases. This characterizes the flow tendency to become more homogeneous, destroying the larger coherent scales.

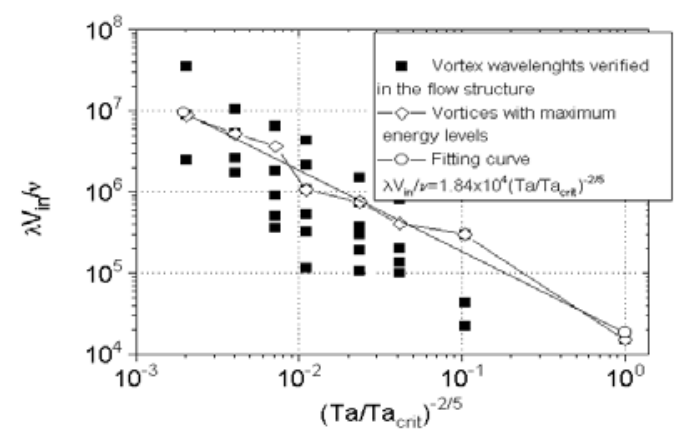

Figure 12. Wavelength measurements of the vortices verified in the TaylorCouette flow structure.

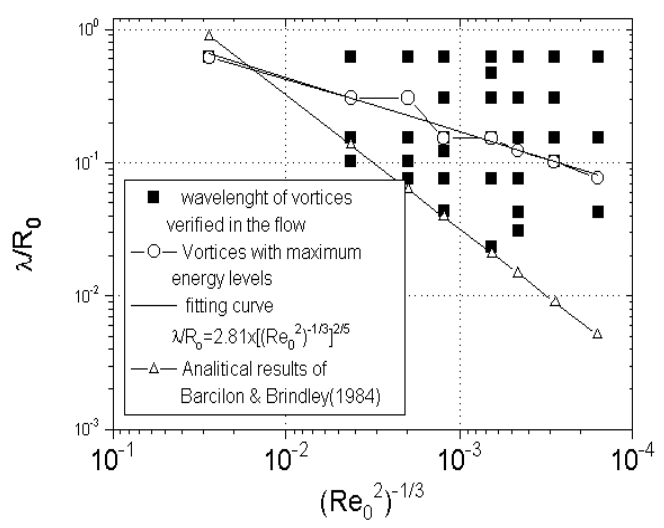

Figure 13. Wavelength measurements of the vortices verified in the protted using the scaling prescribed by Barcilon and Brindley (1984).
As suggest in Wei et al. (1992), because of the Görtler vortices were observed to form at the inner cylinder wall, thus, the scaling proposed by Barcilon \& Brindley (1994), Eq. 1, was not sufficiently general to include results from larger gaps. Therefore, it is presented the Figure 2 that is a plot of vortex wavelengths scaled on innercylinder circumferential velocity and the kinematic viscosity of water versus the ratio $\left(\mathrm{Ta} / \mathrm{Ta}_{\text {crit }}\right)^{-2 / 5}$. The scale wavelength measurements included in this figure are the same data included in the Figure 12. Thus, data of the vortex wavelengths with maximum energy levels in this scaling in interesting way gives a fitting curve as

$$
\frac{\lambda \mathrm{V}_{\text {in }}}{v}=1.84 \cdot 10^{4}\left(\frac{\mathrm{Ta}}{\mathrm{Ta}_{\text {crit }}}\right)^{-2 / 5}
$$

following the $-2 / 5$ power dependence, close to the $-1 / 3$ dependence.

Therefore, one could remark that flow visualization presentation and quantitative scale wavelength measurements suggest strongly that the large scales, as the Taylor cells, influence on the turbulence transition in a large Taylor number range.

\section{Conclusions}

In this study was followed a different procedure to obtain the coherent scale wavelengths present in the flow. In this study, as described above, the coherent scale wavelengths were obtained from the computed spectral density function, that one can regard as a precise procedure to obtain the wavelengths of the coherent scales. However, this is possible only using a technique that allows instantaneous spatial measurements, as the PHANTOMM technique. For example, in Wei et al. (1992), they obtained the Görtler wavelengths measuring the core-to-core separation distance between two counter-rotating vortices in a Görtler vortex pair and by multiplying the core-to-core measurements by two. The measurements were done with the aid of the slow-motion of the video recorder, in this case, when the Görtler vortex was found the video was then frozen and the core-to-core measurements were made. This is a hard procedure to measure the Görtler wavelength and it is passive to introduce significant errors in the measurements.

\section{References}

Barcilon, A., Brindley, J., Lessen, M and Mbbs, F. R., 1979, "Marginal instability in Taulor-Couette flows at a very high Taulor number", J. Fluid Mech. ,94, 453.

Barcilon, A. and Brindley, J., 1984, "organized structures in turbulent Taylor-Couette flow”, J. Fluid Mech., 143, 429.

Benjamin, T. B. and Mullin, T., 1982, "Notes on the multiplicity of flows in the Taulor experiment, J. Fluid Mech., 121, 219.

Biage, M., Harris, S.R., Lempert, W.R. and Smits, A.J., 1996,"Visualization study of Taylor-couette flow", a description of the transition to turbulence, to be published in the 27th AIAA Fluid Dynamics Conference, New Orleans-LA, USA.

Chen, F. J., Malik, M. R. And Beckwith, I. E., 1985, "Ï nstabilities and transition in the wall boundary layers of low-disturbance supersonic nozzles", AIAA, pap. 85, 1573.

Coles, D., 1965, "Transition in circular Couette flow", J. jFluid Mech., 21,385 .

Dahm, W.J.A. and Dimotakis, P.E., 1990, "Mixing at large Schmidt number in the self-similar far field of turbulent jets", J. Fluid Mech., 217, p.299. 
Delery, J. and Coet, M. C., 1992, "Experiments on shock wave/boundary layer interactions produced by a two-dimensional ramp and threedimensional obswtacles", Proc. Workshop on hypersonic flow for rentry problems, Antibes, Berlin Heidelberg hew York: Springer.

Ioss, G., 1986, "Secondaru bifurcations of Taulor vortices and their stability", J. Fluid Mech., 103, 249

Falco, R.E and Nocera, D., 1993,"Quantitative multi-poin measurements and visualization of dense liquid-solid flows using LaserInduced photochemical Anemometry (LIPA)", in: Particulate Two-Phase Flow (ed. M.C.Rocco) Boston: Rutterworth-Heinemann.

Fenstermacler, P. R., Swinney, H. L. and Gollub, J.P., 1979, "Dynamical instabilities and the transition to chaotic Taylor-vortex", J. Fluid Mech., 94 103-128

Foryan, J. M., 1982, "Stability of Görtler vortices in boundary layers", AIAAJ, 20(3), 316

Ginoux, M., 1971, "Streamwise vortices in reattaching high-speed flows: a suggested approach", AIAA, J (, 759-760.

Lempert, W.R., Magee, K., Gee, K.R. and Haughland, R.P., 1995,'Flow tagging velocimetry in incompressible flow using Photo-Activated nonintrusive tracking of molecular motion (Phantom)", Exp. In Fluids, 18 , $249-257$
Mcgray, J.A. and Trentham, D.R., 1989, "Properties and uses of photoreactive caged compounds", Annu. Rev. Biophys. Chem., 18, 239-270.

Rayleich, I., 1916,"On the dynamics of revolving fluids", Scientific Papers, 6, 447-53.

Roberts, G. H., 1965, "Appendix in experiments on the stability of viscous flow between rotating cylinders, VI, Finite amplitude experiments", proc. Roy. Soc., A 283, 531-556.

Smith, G. P. and Townsend, A. A., 1982, "Turbulent Couette flow between concentric cylinders at large Taylor number", J. Fluid Mech., 20, 35. Sparrow, E. M., 1964, "Instability of the flow between rotating cylinders: the wide gap problmem, J. Fluid Mech., 20, 35

Stuart, J.T., 1986,'Taylor-vortex flow: a dynamical system”, SIAM Rev., 28,315 .

Walowit, J. et. Al., 1964,"Stability of flow between arbitrarily spaced concetric cylindrical surface including the effect of a radial temperature gradient”, Trans. ASME E: J. Appl. Mech., 31, 385.

Wei, T., Kline, E. M., K-lee, S. H. and Woodruff, S., 1992, "Görtler vortex formation at the inner cylinder in Taulor-Couette flow", J. Fluid Mech. 245, 47 . 